\title{
Faut-il être ancien pour être légitime? Le conflit des rhétoriques de l'authenticité dans les préfaces de dictionnaires bretons
}

Does it have to be old to be legitimate? Conflicting authenticity rhetoric in the prefaces of Breton dictionaries

\section{Malo Morvan}

\section{OpenEdition \\ Journals}

Édition électronique

URL : https://journals.openedition.org/lbl/298

DOI : $10.4000 / \mathrm{lbl} .298$

ISSN : 2727-9383

Éditeur

Université de Bretagne Occidentale - UBO

\section{Édition imprimée}

Date de publication : 1 mai 2017

Pagination : 239-267

ISBN : 979-10-92331-31-8

ISSN : $1270-2412$

\section{Référence électronique}

Malo Morvan, «Faut-il être ancien pour être légitime? Le conflit des rhétoriques de l'authenticité dans les préfaces de dictionnaires bretons », La Bretagne Linguistique [En ligne], 21 | 2017, mis en ligne le 01 mai 2020, consulté le 30 mars 2022. URL : http://journals.openedition.org//bl/298 ; DOI : https:// doi.org/10.4000/lbl.298

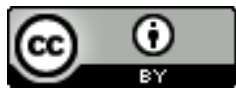

La Bretagne Linguistique est mise à disposition selon les termes de la Licence Creative Commons Attribution 4.0 International. 
Malo MORVAN*

\title{
Faut-il être ancien pour être légitime? Le conflit des rhétoriques de l'authenticité dans les préfaces de dictionnaires bretons
}

\author{
«Il est temps de choisir clairement \\ entre matematik et jedoniezh, \\ entre aritmetik et niveroniezh, \\ entre geometriezh et mentoniezh, \\ entre analis et dezrann / dielfennadur, \\ entre diametr et treuzkiz, \\ entre diagonalenn et treuzvegell, \\ entre problem et kudenn / skoulmad, \\ entre zero et mann, etc. ${ }^{1}$. »
}

\begin{abstract}
Durant tout le $\mathrm{XX}^{\mathrm{e}}$ siècle, des débats se sont engagés à propos du lexique breton. Les participants à ces débats, mêlant bien souvent l'érudition d'une approche linguistique à l'engagement d'un positionnement militant, ont ainsi produit des discours visant à valoriser tel usage linguistique breton par rapport à tel autre concurrent. Parmi les différentes dimensions qui émergèrent dans le débat, nous voudrions nous centrer ici sur les oppositions qui ont visé à promouvoir plutôt tel ou tel usage en invoquant un critère d'« authenticité » : quelle forme linguistique, de celle reconstruite lexicalement à partir de racines dites « celtiques », ou de celle collectée auprès de locuteurs dits « natifs », doit

* Cerlis, Paris 5 Descartes, Rennes 2.

1. Jean Marot, Dictionnaire multilingue des mathématiques, Morlaix, Skol Vreizh, 2002, p. 8.
\end{abstract}


être considérée comme la plus « authentique » ? L’approche proposée ici ne visera pas à répondre à la question, mais à considérer la notion d'« authenticité » comme une référence stratégique permettant aux différents protagonistes de légitimer leur usage en l'ancrant dans un passé valorisé.

Nous commencerons par présenter le cadre théorique qui permet de rendre compte des débats concernés, et notamment quelques notions de sociolinguistique et d'analyse du discours mobilisées pour mener à bien cette analyse, puis nous tenterons de rendre compte de l'émergence de différentes positions dans le débat par les conditions socio-historiques spécifiques au $\mathrm{XX}^{\mathrm{e}}$ siècle, qui ont vu naître une polarisation entre différents profils de locuteurs. Une fois ce cadre posé, nous pourrons observer la manière dont les différents auteurs de dictionnaires bretons justifient les sélections lexicales qu'ils opèrent dans leurs ouvrages, en mobilisant les figures de l'«authenticité» ou de l'«actualité» sous des caractéristiques antagonistes; nous nous concentrerons alors sur les processus discursifs de conversion sémantique par lesquels chaque courant vise à valoriser ses propres choix face à ceux du concurrent.

\section{Position au sein des débats étudiés}

L'objectif de cette contribution est d'analyser des polémiques au sujet des formes linguistiques de la langue bretonne, et non de défendre l'une ou l'autre des thèses mises en avant par leurs protagonistes. En particulier, il s'agit d'analyser la manière dont est invoquée l'idée d'une «authenticité » de certains usages afin de valoriser un certain type d'usages linguistiques contre un autre, au sein d'un cadre concurrentiel entre des tendances militantes divergentes. Nous nous intéresserons en particulier à des débats relativement récents, portant globalement sur deux tendances : l'une, pour laquelle l'«authenticité » du breton s'évalue essentiellement sur une base étymologique et lexicologique, par l'absence de termes ressemblants au français, l'autre, pour laquelle « authentique » signifie plutôt qu'un usage a été collecté auprès de locuteurs souvent désignés comme «natifs », et estimés à ce titre plus fiables que d'autres nommés « néo-locuteurs ». 
Le but d'une telle analyse n'est pas de déclarer quel type d'usage serait plus « authentique » qu'un autre, il s'agit plutôt, d'une part, de revenir sur les présupposés partagés en commun par les tenants des différentes positions (notamment l'idée que l'usage du mot " authentique » pour désigner des pratiques linguistiques veut dire quelque chose), d'autre part, de comprendre les raisons qu'a chacun, premièrement, de faire usage de cette notion d'«authenticité », deuxièmement de tenir pour « authentique » ceci plutôt que cela (étymologie-lexique-morphologie dérivationnelle d'un côté, syntaxe-phonologie-phonétique-prosodie de l'autre ; une filiation établie envers d'autres langues « celtiques » d'un côté, le témoignage de locuteurs estimés être des informateurs fiables de l'autre, etc.).

Le fait que nous ne prenions pas position au sein de ces débats n'implique pas la croyance illusoire en une quelconque forme de « neutralité » dans la recherche : le propos tenu ici n'est pas neutre du tout, puisqu'il remet en question les présupposés partagés par les uns et les autres (ce qui peut être une bonne manière de se mettre tout le monde à dos...).

\section{Des présupposés à réinterroger}

Parmi ces présupposés, ceux que nous remettrons en question seront notamment : d'une part, la validité théorique de la notion de «langue » en tant que concept descriptif ${ }^{2}$, d'autre part, la pertinence de la notion d'« authenticité ». Nous expliquerons ce second point par deux ensembles de raisons :

2. Le cadre de cet article n'offre pas la place pour en expliquer les raisons. Nous devons nous contenter de renvois bibliographiques envers des ouvrages de différentes traditions sociolinguistiques au sein desquelles cette remise en question est déjà établie : Pierre Bourdieu, Langage et ponvoir symbolique, Paris, Seuil, 2001 ; Michel PÊCHeux et Françoise GADET, La langue introuvable, Paris, Maspero, 1981 ; Jean-Michel ELOY, La constitution du picard. Une approche de la notion de langue, Louvain-la-Neuve, Peeters, 1997 ; Louis-Jean CALVET, Essais de linguistique. La langue est-elle une invention des linguistes ?, Paris, Plon, 2004 ; Cécile CANUT, Une langue sans qualité, Limoges, LambertLucas, 2007 ; Sinfree MAKONI et Alastair PENNYCOOK, Disinventing and reconstituting languages, Clevedon, Multilingual matters, 2006 ; Patrick SÉRIOT, Les langues ne sont pas des choses. Discours sur la langue et souffrance identitaire en Europe centrale et orientale, Paris, Petra, 2010. 
Historiquement, la notion d'« authenticité » suppose une certaine fixité entre les usages actuels et des usages plus anciens, alors que ceux-ci évoluent en permanence : même les usages linguistiques d'aujourd'hui tenus pour « authentiques » n'ont rien à voir avec le breton d'un Jehan Lagadeuc ou d'un Guillaume Quiquer. Par ailleurs, le breton de ces mêmes auteurs, rétrospectivement catégorisé comme du «brez̧honeg beleg » («breton de curé »), ou « jargon mixte » par La Villemarqué ${ }^{3}$ a pu être considéré comme « inauthentique » par rapport à un âge d'or rêvé sans contact avec une autre langue. Enfin les usages linguistiques en breton du XXIII siècle pourront sûrement être considérés comme « inauthentiques » par rapport aux nôtres (sauf si l'on a abandonné la notion - ou les usages - entre-temps).

Sociolinguistiquement, nous disposons depuis plusieurs décennies de tout un attirail de notions qui permettent de rendre compte des situations de circulation entre les usages linguistiques et qui rendent poreuses les catégorisations au sein desquelles on les enferme généralement : notamment celles de « contact de langues » ou « interférences linguistiques » depuis Uriel Weinreich ${ }^{4}$, « code-switching» et « code-mixing», surtout depuis John Gumperz ${ }^{5}$, «véhicularisation », qui me semble apparue depuis l'étude des marchés en Afrique, dans les années 1990, « interlangue ${ }^{6}$ », et «calque syntaxique », ou encore "colinguisme» depuis Renée Balibar ${ }^{7}$ (cette dernière me semblant particulièrement utile - plus que celle de «bilinguisme »- pour désigner l'époque où les évêchés avaient un usage parallèle de l'écriture en breton et en français), etc.

Sans vouloir discuter ici de la pertinence de chacune de ces notions, cette énumération suffit à montrer qu'il est possible de proposer

3. Théodore Hersart de LA VILLEMARQué, Essai sur l'bistoire de la langue bretonne, 1847, dans l'édition critique donnée par Bernard Tanguy, Aux origines du nationalisme breton, tome 2, Paris, Union Générale d'Éditions, 1977, p. 57.

4. Uriel WeInREICH, Languages in contact. Findings and problems, New York, Linguistic Circle of New York, 1953.

5. John GUMPERZ, Engager la conversation. Introduction à une sociolinguistique interactionnelle, Paris, Minuit, 1989.

6. Enrico ARCAINI et Bernard Py, Interlingua. Aspetti teorici e implicazioni didattiche, Rome, Istituto della Enciclopedia italiana fondata da G. Treccani, 1984.

7. Renée BALIBAR, Le colinguisme, Paris, Presses Universitaires de France, coll. « Que saisje ?», 1993. 
une approche descriptive ou explicative des phénomènes d'évolution linguistique menant certains à considérer que des usages deviennent « inauthentiques» (particulièrement, l'influence de la langue française, sous une forme ou sous une autre), plutôt que de formuler un jugement axiologiquement marqué à l'égard de ces phénomènes. Il sera donc possible de revenir de et sur les discours dénigrant certains usages sous prétexte d'« inauthenticité »: d'une part, remplacer ces discours par une analyse qui tente d'expliquer, socio-historiquement, l'émergence de tels usages plutôt que de les condamner au nom d'une conception implicite de la langue rarement remise en question, dominée par la hantise de la ressemblance au français, d'autre part, analyser les raisons de l'émergence de tels discours d'authenticité et de démarcation envers le français. Parmi les usages concernés par ces discours et qui peuvent être analysés à nouveaux frais, nous trouverions, pour les uns, l'usage de termes comme «an tele» ou «an trakteur», généralement par les personnes âgées de milieu rural, pour les autres, un ensemble d'usages syntaxiques, phonologiques, phonétiques, et prosodiques, qui témoigneraient de l'influence du français.

La situation de « contact linguistique » entre breton et français va dans les deux sens ${ }^{8}$, bien que d'une manière asymétrique en raison de la relation d'inégalité entre les deux ensembles d'usages linguistiques, et elle peut s'expliquer par des raisons historiques : jusqu'au début du $\mathrm{XX}^{\mathrm{e}}$, les usages écrits émanaient essentiellement de religieux ${ }^{9}$ formés aux humanités classiques (latin, français, grec), et les usages du breton servaient en grande partie (chez un Michel Le Nobletz, Julien Maunoir, Claude-Guillaume Marigo, Charles Le Bris, etc.) à diffuser localement un ensemble de thèses dont le vocabulaire s'était élaboré depuis des siècles par des discussions théologiques sur des bases latines. Au cours $\mathrm{du} \mathrm{XX}^{\mathrm{e}}$ siècle, des changements tant politiques qu'économiques menèrent à accroître les échanges avec le reste de la France et à associer la langue française aux nouveaux cadres de pratiques, et une certaine

8. Comme nous le rappelle le récent succès des ouvrages d’Hervé Lossec concernant les bretonnismes.

9. Yves Le Berre, La littérature de langue bretonne. Livres et brochures entre 1790 et 1918, Brest, Emgleo Breiz, 1994. 
génération considéra alors sa transmission comme plus opportune sur un marché de l'emploi en pleine reconfiguration ${ }^{10}$.

C'est ainsi que lorsque des personnes traitant de la langue bretonne, y compris, voire surtout linguistes, sociolinguistes, historiens de la langue, étymologistes, se prononcent d'une quelconque manière sur un éventuel caractère « inauthentique » de ces situations de contact de langue, ils se situent eux-mêmes, de manière plus ou moins consciente, dans une posture qui est tout autre que celle d'une explication : il s'agit de se référer à un usage linguistique que, dans un cadre de concurrence au sein des milieux de militantisme linguistique, on souhaite valoriser contre un autre ${ }^{11}$. Or, comme nous l'avons dit plus haut, la remise en question de notions comme celle de "langue » ou d'«authenticité » constitue le point de départ et non le but de notre étude : il s'agit surtout d'analyser de quelle manière une notion comme celle d'« authenticité » pourra se trouver mobilisée dans des discours de valorisation de certains usages linguistiques au sein d'un cadre concurrentiel.

\section{Quels débats?}

On peut expliquer les débats des dernières décennies concernant la langue bretonne si l'on les met en rapport avec les évolutions historiques et sociales du $\mathrm{XX}^{\mathrm{e}}$ siècle qui ont engendré une polarisation des locuteurs du breton en deux ou trois profils :

- d'une part, des locuteurs qui pratiquaient des usages localisés de la langue, quotidiennement et oralement, surtout dans un milieu rural, et qui ont vu l'abandon progressif des conditions d'un tel usage ;

- d'autre part, des intellectuels et militants qui se sont réapproprié la langue dans une démarche volontariste, qui en connaissent des usages écrits et l'investissent d'un projet politique ;

10. Fañch Broudic, La pratique du breton de l'Ancien Régime à nos jours, Rennes, Presses Universitaires de Rennes, 1995.

11. De là surgit une ambiguïté constitutive à tout mon travail de recherche sur ces débats, puisque la limite entre «sources primaires» et «secondaires» s'abolit, les auteurs produisant des analyses auxquelles je me réfère peuvent aussi bien, quelques lignes plus loin, produire des jugements de hiérarchisation qui me les rendent intéressantes cette fois en tant que corpus à étudier. Ceci vaut tout particulièrement pour des auteurs qui ne mâchent pas leurs mots, comme Christian Joseph Guyonvarc’h ou Martial Ménard. 
- on pourrait ajouter un troisième profil qui serait celui des nouveaux locuteurs issus des structures mises en place par les seconds, mais qui ne se situent pas forcément eux-mêmes dans une démarche militante (élèves des écoles et cours du soir, etc.).

En reprenant et détournant quelques débats de dénomination à propos du breton, et quelques propositions de typologies déjà existantes, je proposerais d'appeler « bretonnants » les premiers, « bretonnantistes » les seconds, et « brittophones » les troisièmes. On pourrait expliquer la différence dans le rapport au langage entre les deux premiers groupes de locuteurs en se reposant sur les travaux de Bernard Lahire $^{12}$, et la distinction qu'il propose entre, d'une part, un rapport pragmatique au langage en situation d'interlocution, et d'autre part, un rapport nommé «scriptural-scolaire », nourri par la systématicité de l'approche écrite, et manipulant réflexivement des énoncés pris pour eux-mêmes et non pour ce qu'on leur fait dire en interaction.

Il convient toutefois de remarquer que ce ne sont pas ces profils de locuteurs que l'on retrouve directement dans les débats linguistiques, pour plusieurs raisons. Tout d'abord parce que de tels débats sur la langue, nécessitant un rapport réflexif au langage, n’impliquent donc généralement pas les locuteurs de la première catégorie : on trouve chez eux éventuellement des réflexions comme "n'eo ket memes brę̧boneg » (c'est pas le même breton), ou des indications sur la zone où l'on parlerait un breton estimé meilleur, mais on ne trouve pas vraiment, chez les locuteurs âgés de milieu rural qui ont appris le breton dans leur famille et qui l'ont utilisé au quotidien pour désigner les réalités de leur vie, des prises de position quant à savoir si «exponentiel le» doit être traduit par «argemmvac'hek» ou par «eksponantel», ou s'il y a lieu de distinguer dans l'orthographe entre les fricatives

12. Bernard LAHIRE, Culture écrite et inégalités scolaires. Sociologie de l'« échec scolaire »à l'école primaire, Lyon, Presses Universitaires de Lyon, 1993. Lahire s'appuie lui-même essentiellement sur Jack GOODY (La raison graphique. La domestication de la pensée sawvage, Paris, Minuit, 1979) pour l'influence de l'écriture sur les modes de pensée et Mikhail BAKHTine (ou Valentin Volochinov, Le marxisme et la philosophie du langage. Essai d'application de la méthode sociologique en linguistique, Paris, Minuit, 1977 [1929]) pour les effets de décontextualisation du message linguistique appliqués par la linguistique saussurienne, dont il considère qu'elle s'applique également à l'enseignement. 
vélaires sourdes et voisées. Par ailleurs, ces débats ont généralement lieu sur des supports écrits fortement codifiés où l'expression est soumise à une relative sélection (Bourdieu parlerait d'un "marché restreint » où le " prix » d'entrée est élevé) : revues littéraires, militantes et scientifiques, livres (qui écrit des dictionnaires ?), etc. Il se pourrait que des contributions de locuteurs du premier profil soient rejetées dans un tel cadre, mais il n'y a généralement pas besoin d'arriver à ce genre de situation : encore faut-il que les lieux des débats, leur teneur, soient connus de tels locuteurs, qu'ils souhaitent dire quelque chose à ce sujet et qu'ils se sentent la confiance nécessaire pour se lancer dans un débat. Dans la mesure où ces conditions sont rarement réunies (ce qui ne veut pas dire « jamais »), il faut prendre en compte que les débats concernant la « qualité » du breton, et notamment son « authenticité », sont essentiellement des débats d'intellectuels, y compris du côté de ceux qui se situeront en représentants d'usages dits " populaires ».

Il convient par conséquent de dédoubler la typologie des profils de locuteurs par une seconde, portant sur les positions défendues. On peut pour cela commencer par remplacer la notion de "bretonnant» par celle de " pro-bretonnant», qui désigne des auteurs défendant les positions des bretonnants, mais ayant eux-mêmes le rapport scripturalscolaire au langage leur permettant d'exprimer leur position en formulant des propos métalinguistiques systématisés dans des écrits fortement sélectifs. On pourra alors désigner deux pôles de positions au sein des débats :

- Les « continuistes », qui correspondent aux «pro-bretonnants » que nous venons de mentionner, sensibles à la variété dialectale qu'ils considèrent comme une richesse, mettent en avant une pratique du breton qui viserait à renouer la continuité intergénérationnelle envers les « bretonnants».

- Les « refondateurs", sensibles au développement d'une littérature, d'institutions, d'une reconnaissance officielle de la langue, souhaitent se doter d'outils politiques pour revaloriser, aux yeux d'une approche scripturale-scolaire de la langue, un usage qu'ils estimeraient confiné à la quotidienneté et à une forme de trivialité. On trouve généralement dans cette tendance des positions témoignant d'un certain élitisme linguistique, et d'un nationalisme politique. 
Il convient de présenter quelques précautions quant à ces classifications $^{13}$ :

Premièrement, si nous sommes partis d'une remise en question de la notion de "langue », et d'autres classifications simplistes qu'elle suppose (notamment celle de « dialecte»), il ne faut pas que ce soit pour retomber dans d'autres classifications tout aussi réductrices concernant les positions des locuteurs. Ainsi, pour éviter de tomber dans un travers labovien qui consisterait à délimiter à l'avance des groupes de locuteurs pour ensuite expliquer leurs productions par rapport à la conformité dont elles témoignent envers les groupes préconstruits, il faut bien avoir pour précaution de prendre les catégories de locuteurs («bretonnants », «bretonnantistes », « brittophones ») comme des idéaux-types, au sens où l'a défini Max Weber ${ }^{14}$, c'est-à-dire, des fictions théoriques, dont on sait pertinemment qu'aucune personne n'y correspond complètement en raison de la complexité des situations sociales, mais qui permettent d'ajouter un peu de clarté dans les phénomènes observés en dessinant schématiquement des grands traits ${ }^{15}$.

13. Dans la mesure où ces typologies sont formulées dans le cadre d'un travail de thèse en cours de rédaction, elles sont ici soumises à discussion. Elles impliquent aussi de jeter un regard critique sur les catégories souvent employées dans les débats, comme celles de «locuteurs natifs » («native speakers»), « terminal speakers», « locuteurs traditionnels »,

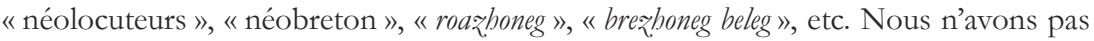
le temps de discuter chacune de ces catégories, disons juste rapidement que leurs connotations axiologiques impliquant déjà une prise de position, elles ne peuvent pas être reprises pour l'analyse d'un tel débat.

14. «Si l'on veut penser quelque chose d'univoque sous ces termes, la sociologie est obligée d'élaborer de son côté des types ("idéaux") "purs" de chacune de ces sortes de structures qui révèlent alors chacune pour soi l'unité cohérente d'une adéquation significative aussi complète que possible, mais qui, pour cette raison, ne se présentent peutêtre pas davantage dans la réalité sous cette forme pure, absolument idéale, qu'une réaction physique que l'on considère sous l'hypothèse d'un espace absolument vide. Ce n'est que sur la base de ce pur type ("idéal”) qu'une casuistique sociologique est possible.» (Max Weber, Économie et société, Paris, Plon Pocket Agora, 1995 [1922], p. 49.)

15. On pourra bien entendu s'intéresser tout particulièrement à des locuteurs qui ne rentrent pas dans ces catégories : Loeiz Herrieu ou Añjela Duval en fourniraient un bon exemple, mais aussi des locuteurs qui auraient commencé à apprendre le breton dans un cadre scolaire ou universitaire (des «brittophones») puis auraient décidé d'aller le réapprendre par un travail de collectage, et éventuellement en allant s'immerger dans les modes de vie ruraux et les travaux des champs, pour se rapprocher de la langue des «bretonnants ». 
Deuxièmement, concernant les deux types de positions défendues dans les débats, il faut également bien considérer qu'elles ne sont pas données d'avance, mais résultent de constructions et de reconfigurations complexes propres au contexte des échanges polémiques, on peut décrire leur émergence en mentionnant les processus de « dichotomisation » et de « polarisation » tels qu'ils sont définis par Ruth Amossy ${ }^{16}$.

\section{Présupposés communs dans ces débats}

Si tout débat implique certes un désaccord, il implique néanmoins aussi que les protagonistes s'accordent sur les termes mêmes dans lesquels la question se trouve posée ${ }^{17}$. Ici en l'occurrence, chacun des débats s'appuie, à titre de topos $^{18}$, sur un ensemble de présupposés communs, que l'on pourrait formuler ainsi : «ancien = légitime »; " influence du français = récent = illégitime », et la différence résidera dans le critère tenu pour pertinent pour l'observation de cette «influence du français» dans la langue. Celui-ci sera investi d'un contenu différent selon les deux tendances :

- pour les bretonnantistes, c'est dans le lexique, et notamment dans son étymologie, que s'observera l'(in)authenticité des usages, et la

16. Ruth Amossy, Apologie de la polémique, Paris, Presses Universitaires de France, 2014, p. 55-61. Le premier processus explique la manière dont la complexité d'un grand nombre de questions se trouve progressivement schématisée sous la forme d'une opposition "pour/contre », « noir/blanc ». Le second explique la manière dont des groupes de locuteurs de profils très différents en viennent, au sein d'un débat précis, à reconfigurer le jeu habituel de leurs oppositions pour se trouver à communier dans une réponse partagée. Si Amossy prend l'exemple du débat sur le voile, qui vit des positions communes défendues par des religieux musulmans, des féministes, des membres d'associations de quartiers, des historiens, etc., le cas breton est intéressant également, voyant singulièrement communier des religieux et des communistes dans la défense des positions " pro-bretonnantes ».

17. Pierre Bourdieu, Langage et pouvoir symbolique, Paris, Seuil, 2001, p. 89.

18. En analyse du discours, on appelle «topos» un lieu commun tenu pour partagé auprès du public auquel on s'adresse, sur lequel il est possible de s'appuyer en accrochant sa propre thèse, ou en reconfigurant la caractérisation d'une entité discutée à partir d'associations d'idées déjà répandues dont elle sera rendue parallèle. Voir Jean-Claude Anscombre, Théorie des topoï, Paris, Kimé, 1995 ; Jean-Claude Anscombre et Oswald DuCROT, L'argumentation dans la langue, Bruxelles, Mardaga, 1983 ; Ruth AMOssy et Anne Herschberg Pierrot, Stéréotypes et clichés, Paris, Armand Colin, 2014. 
morphologie dérivationnelle sera abondamment mobilisée pour produire des usages « authentiquement» celtiques ;

- pour les pro-bretonnants, ce sont des propriétés telles que la syntaxe, la phonologie, phonétique et prosodie, qui se révéleront pertinentes pour l'observation de tels phénomènes, et c'est la conformité avec les locuteurs décrits comme «natifs » qui fournira le point de comparaison ${ }^{19}$.

On peut se demander s'il est possible de fournir une explication des raisons pour lesquelles les uns valorisent telle dimension linguistique, et les autres, telle autre. Or, il nous semble qu'une telle répartition puisse s'expliquer, si l'on reprend avec Lahire la caractérisation des différents rapports au langage, l'un que l'on pourrait nommer " pragmatique-interlocutif», l'autre «scriptural-scolaire». En mettant les débats en rapport avec les modes d'apprentissage et de pratique quotidienne du breton de chaque profil d'interlocuteurs, il est possible d'expliquer comment les cadres de pratique de chacun incitent les uns à percevoir la langue comme essentiellement faite de lexique, d'étymologie, et de morphologie, et les autres, comme faite de syntaxe, phonologie, phonétique, et prosodie.

Les «bretonnantistes » ont appris la langue par l'intermédiaire de dictionnaires, grammaires ou de cours où se trouvaient explicitées systématiquement et métalinguistiquement les propriétés d'une langue avant qu'ils ne la parlent couramment (tableau des mutations consonantiques initiales, table des conjugaisons des verbes, etc.), leur engagement politique les a poussés à souhaiter marquer une certaine démarcation envers les usages français, ainsi qu'à vouloir montrer que la langue bretonne était «capable » de s'exprimer dans tous les domaines, y compris les plus « nobles » (littérature (Gwalarn), géométrie (Célestin Lainé), psychanalyse (Guy Étienne), philosophie (Tugdual Kalvez), etc.), sans avoir recours à un substrat identifié comme «français». Par ailleurs, ces contextes d'écriture sont ceux où, en breton comme dans d'autres

19. On trouvera dans les débats des analyses d'autres phénomènes linguistiques, notamment la sémantique verbale ou l'usage des prépositions, mais ils ne semblent pas spécifiques à l'une des approches en particulier; leur analyse dépend, au cas par cas, du déroulement de l'argumentation de chaque auteur. 
langues, on vérifie dans les dictionnaires et grammaires la justesse des termes et des expressions. Qu'il s'agisse du mode d'apprentissage, de l'engagement militant, ou des contextes de pratique, on conçoit que ces dimensions convergent vers une description de la langue où prédomineront les approches lexicologique, étymologique, et morphologique, qui ont en commun d'aborder les formes linguistiques pour elles-mêmes, hors de tout contexte d'interlocution, afin d'en analyser le sens, l'histoire, ou d'éventuels rapports de systématicité (paradigmes, flexions, dérivations).

Les «bretonnants», défendus par les "pro-bretonnants», ont appris le breton dans leur enfance, dans un milieu familial, au sein d'interactions orales quotidiennes, pour désigner des réalités pratiques au sein de conversations en face-à-face. La langue devant servir à dire le monde environnant, à interagir avec d'autres locuteurs, ils purent changer de registre linguistique lorsque les transformations socioéconomiques rendirent plus opportuns, d'un point de vue pratique, les échanges en français. On comprend également qu'un tel mode d'apprentissage et contexte de pratique pousse ces locuteurs, ou leurs défenseurs, à percevoir la langue essentiellement sous ses propriétés plus directement liées aux échanges pratiques à l'oral : ce que l'on prononce (phonologie, phonétique, prosodie), la manière dont on construit les phrases (une syntaxe plus orientée vers l'efficacité pratique de l'acte de communication, où les termes importants sont placés en début de phrase, que vers le souci d'un style conforme à des règles édictées).

Chacun des contextes d'apprentissage et de pratique des différents profils de locuteurs nous semble donc à même de rendre compte des raisons pour lesquelles les uns perçoivent la langue essentiellement comme faite de lexique, morphologie, étymologie, propriétés saillantes d'un rapport scriptural-scolaire à la langue, et les autres, comme faite de prononciation et de formation de phrase, nécessités immédiates de toutes utilisations orales d'une langue en situation d'interlocution.

On le voit, une telle caractérisation n'a pas pour but d'ériger l'un des usages comme supérieur ou inférieur à un autre. Au contraire, elle vise à rendre compte des raisons qui gouvernent les désaccords concernant les usages linguistiquement légitimes, en les renvoyant aux conditions de socialisation langagière de chaque grand profil de locuteurs (une fois prises toutes les précautions nécessaires pour se garder de réifier ces 
profils). À partir de là, il devient possible de retourner la perspective à propos d'une notion comme celle d'«authenticité » : il s'agira, non plus de la manier au premier degré en tant que concept justifiant une quelconque supériorité, mais bien de revenir sur ses usages, c'est-àdire d'observer de quelle manière elle est utilisée au sein des discours de légitimation de certaines pratiques linguistiques dans un espace discursif marqué par une concurrence entre ces deux tendances de promotion du breton.

\section{La fonction sociale des dictionnaires}

Il nous reste maintenant à voir en quoi les préfaces de dictionnaires peuvent constituer des lieux particulièrement stratégiques pour l'expression de tels discours concurrentiels d'authentification des usages linguistiques. Pour cela, nous devons nous poser une question : que produit, socialement, un dictionnaire? Ceux-ci ont pour conséquence, lorsqu'ils sont diffusés et qu'un crédit suffisant leur est accordé, de consacrer certains usages linguistiques en leur conférant une certaine officialité : à ce titre, on peut les considérer comme une certaine forme d'institution, qui déclare quels mots sont considérés comme appartenant à la langue et lesquels, n'y figurant pas, doivent être tenus pour «périphériques » ou « marginaux ».

Or, un dictionnaire ne peut pas contenir tous les mots du lexique. Certes, en ce qui concerne le cas breton, le peu de moyens, tant financiers qu'humains, rend déjà beaucoup plus difficile de rédiger et éditer des ouvrages volumineux, mais le problème n'est pas là : il se situe fondamentalement dans l'écart entre, d'une part, les productions orales quotidiennes de groupes de locuteurs hétéroclites, marquées par plusieurs facteurs de diversification (que l'école variationniste listerait en : variation diachronique (liée à l'évolution de la langue), diatopique (liée aux territoires), diastratique (liée aux milieux sociaux), diaphasique (liée aux conditions d'interlocution), etc.), et d'autre part, une liste de mots qui se trouvera toujours trop restreinte pour couvrir la totalité de ces usages et de leurs renouvellements permanents. Ce constat quant au caractère nécessairement sélectif de tout dictionnaire, soulève alors deux questions : 
D'une part, selon quels critères les auteurs choisissent-ils d'inclure ou non certains termes?

D'autre part, quels effets ce choix produit-il quant à la manière dont la langue se trouve représentée?

On peut ici proposer une comparaison avec la situation du français : tous les ans, les médias s'emparent des apparitions et disparitions de lexique dans le Robert ou le Larousse: si «boloss » ou «kiffer» entrent dans le dictionnaire, alors on pourra considérer qu'ils ont accédé à une forme de reconnaissance institutionnelle. Par ailleurs, on entend parfois certaines personnes qui, après avoir prononcé un mot, se demandent si «cela se dit» ou si le mot « existe ». Qu'est-ce qu'une telle question pourrait bien vouloir dire de la part de quelqu'un qui doit être bien informé de l'existence du mot en question, venant de le prononcer? Ce genre de questions repose en fait sur l'idée selon laquelle certains mots ont besoin d'une consécration officielle pour avoir droit de cité, et pour pouvoir être dits véritablement « exister ». Ainsi, si un mot entre dans un dictionnaire, c'est qu'il « existe réellement», car il aura été reconnu digne d'y figurer par des spécialistes du lexique.

Pourtant, la comparaison avec la situation française s'arrête là, car dans le cas du français, un gros travail d'institutionnalisation a produit un relatif consensus quant à la définition de « la langue » (sauf chez les sociolinguistes) : la reconnaissance accordée au Larousse ou au Robert est assez unanime ${ }^{20}$. En breton, on ne peut pas considérer actuellement qu'il existe une telle unanimité, précisément en raison de la situation de concurrence pour l'accès à une reconnaissance entre divers courants du militantisme breton qui proposent chacun des définitions différentes de la langue. Cette concurrence pour l'accès à l'officialité a été vive à propos des orthographes, mais elle existe aussi pour le lexique. Un des lieux stratégiques de la formulation d'une telle concurrence est la formation de nouveaux locuteurs. L'Ofis ar brezhoneg dispose d'une certaine officialité, surtout depuis qu'il est devenu public, mais celle-ci

20. On trouverait d'autres ouvrages considérés comme canoniques : le dictionnaire de l'Académie, le TLFI, et certains ouvrages selon les disciplines concernées (le dictionnaire de Lalande pour la philosophie, celui de Laplanche et Pontalis pour la psychanalyse, etc.). 
n'est pas encore implantée et reconnue partout (nous connaissons des milieux où l'on dit : «Polis ar brę̧oneg »).

Schématiquement, on pourrait dessiner deux grandes tendances chez les lexicographes contemporains du breton:

D'un côté, on profite d'éditer un dictionnaire pour forger de nouveaux mots, proposés aux apprenants, en espérant leur diffusion : ceuxci portent en général sur des savoirs techniques et spécialisés, et se situent généralement dans une démarche d'emprunt à des langues dites " celtiques », ou à des formes anciennes du breton. On trouve les travaux de $S A D E D$, Preder, Imbourc'h, parmi les plus représentatifs de ce courant, mais celui-ci englobe plus largement la tendance gwalarnienne, on pourrait y ranger également Martial Ménard, Pêr Denez, le dictionnaire de philosophie par Tugdual Kalvez, etc. ${ }^{21}$.

D’un autre côté, on choisit de représenter dans un dictionnaire les termes tels qu'ils sont collectés, c'est-à-dire que l'on espère produire un dictionnaire représentatif d'usages oraux attestés parmi des locuteurs que l'on investit comme plus fiables que d'autres. On pourrait considérer les travaux de Jules Gros ${ }^{22}$ comme fondateurs de ce courant, et y situer des ouvrages comme le dictionnaire Garnier (et sa réédition sous le nom d'Andreo Ar Merser), celui de Francis Favereau (même s'il a élargi ses références aux œuvres littéraires), Le Trégorrois à Plougrescant de Jean Le Dû, les travaux de Daniel Giraudon ou Jean-Yves Plourin, etc.

Ici encore, il ne s'agit pas de donner raison ou tort à l'une ou l'autre des tendances (ce qui est souvent attendu par des chercheurs

21. On pourrait vouloir faire remonter cette tendance à Jean-François Le Gonidec et Théodore Hersart de La Villemarqué (voir jusqu'à Louis Le Pelletier) puis prendre en compte la contribution importante de Frañsez Vallée dans cette démarche; pourtant il faut faire attention à l'anachronisme, dans la mesure où, si ces auteurs ont fourni des jalons qui inspirèrent leurs successeurs, il importe de prendre en compte les évolutions dans la pratique du breton et dans le militantisme, qui ont créé le contexte particulier où émergent les ouvrages de lexicographie d'après-guerre.

22. Ici, il faudrait prendre en compte que l'influence du travail de Jules Gros, souvent considéré comme fondateur pour les auteurs de ce courant, a eu pour conséquence d'orienter une telle recherche, non seulement vers la lexicographie, mais aussi vers la stylistique, et le collectage d'expressions populaires, proverbes, etc. De même que pour l'autre courant : on pourrait encore chercher des «précurseurs », mais ce serait négliger les évolutions historiques qui ont engendré un contexte spécifique pour l'édition des ouvrages dans les dernières décennies. 
bretons eux-mêmes engagés dans ces débats), mais bien plutôt de tenter d'expliquer cette dichotomie en la renvoyant aux conditions d'apprentissage, de pratique et d'engagement militant de chacun de ses membres.

\section{La conversion des valeurs}

Chacun des dictionnaires ainsi cités intègre et exclut des formes lexicales, selon un faisceau de critères déterminé par la tendance au sein de laquelle il se situe. Pour les uns, il s'agit d'exclure des mots identifiés comme français ${ }^{23}$, pour les autres, des formes lexicales considérées comme des inventions d'intellectuels, qui ne seraient pas attestées chez des locuteurs jugés plus fiables.

Dans les deux cas, le choix s'opère sur la base d'un critère d'exclusion qui repose sur la définition d'un usage jugé plus «véritable» ou « authentique»: on retrouvera alors, dans les justifications, des mentions comme celle d'un « esprit » ou « génie de la langue », qui contribuent à la réifier, pour des besoins stratégiques de positionnement concurrentiel.

Dans les discours justifiant ces choix lexicaux (notamment les préfaces de dictionnaire), on observe en fait une valorisation sur deux fronts : la légitimité d'un usage à promouvoir s'assoit sur une authenticité revendiquée, mais aussi sur la mention d'une actualité, d'une correspondance des formes lexicales proposées avec les besoins linguistiques d'aujourd'hui. La pratique concurrente peut ainsi se trouver

23. Il est important ici de préciser «identifiés comme français» ou «ressemblant au français », car il est plus souvent question d'étymologie populaire reposant sur les proximités directement observables, que de réelles constructions étymologiques, qui exigeraient une solide connaissance du latin et de l'ancien français pour percevoir toutes les influences anciennes non reconnaissables immédiatement, notamment par l'effet de mots aujourd'hui désuets en français, mais dont des appropriations se sont conservées dans l'usage breton. On voit par exemple certains militants préférer «ar roched» à "ar chemisenn», ou «ar gazetenn» à «ar journal», car les seconds termes de chaque couple seraient trop ressemblants à des formes françaises contemporaines, alors que les premiers proviennent aussi de formes françaises plus désuètes comme «rochet / rochette» (vêtement porté par des religieux), et «gazette». L'introduction au Dictionnaire étymologique de Christian Joseph GUYONVARC'H (Dictionnaire étymologique du breton ancien, moyen et moderne. Origine et histoire des mots, Rennes, OGAM-Celticum, 1973) est instructive sur ce point, même s'il faut pouvoir se détacher de son propos souvent très polémique. 
renvoyée symétriquement, non seulement à une forme d'invention récente, (adoption d'un vocabulaire francisé depuis quelques décennies pour les uns; invention de termes par des intellectuels pour les autres), mais aussi, et en même temps, à une forme de passéisme (d'un côté, vocabulaire de la vie rurale non adapté aux locuteurs urbains d'aujourd'hui ; de l'autre, enfermement dans un lexique celtique qui ne permet pas les communications à l'international, reposant sur des langues latines).

On pourrait se contenter de considérer comme contradictoires des discours qui revendiquent à la fois une ancienneté sous la forme d'une « authenticité » et une actualité, et qui tentent de discréditer les usages concurrents à la fois comme relevant d'une invention récente et comme témoignant d'un archaïsme. Pourtant, il nous semble plus fructueux de mobiliser ici l'analyse de discours, et particulièrement l'analyse des processus de conversion sémantique telle qu'elle a été présentée par Dominique Maingueneau ${ }^{24}$ pour rendre compte du phénomène. Il s'agit d'observer comment certaines notions se trouvent investies de connotations positives ou négatives dans le contexte d'une polémique en fonction des différents réseaux conceptuels au sein desquels chacun des camps les fait figurer ${ }^{25}$. Pour Maingueneau, ce processus aboutit à une « grammaire de l'incompréhension » : deux systèmes théoriques, chacun cohérent et soudé, ne peuvent que réécrire dans leur réseau sémantique les valorisations/dévalorisations du propos opposé, pour se les réapproprier. Ce phénomène aboutit au fait que ce que dit l'un n'est jamais ce qu'en entend l'autre, car chacun l'entend au sein de son

24. Dominique Maingueneau, Sémantique de la polémique, Lausanne, L'Âge d'Homme, 1983.

25. Maingueneau, analysant les polémiques entre l'« humanisme dévot » et le jansénisme au XVII siècle, observe que chaque courant réinterprète le propos de son opposant au sein d'un réseau sémantique de significations, d'association d'idées, et d'évidences, qui le convertit littéralement au sein de son propre système d'identifications et de valorisations. Il prend l'exemple du mot «doux», qui signifie pour les premiers l'harmonie divine, la contemplation du beau monde que Dieu a créé et où il se trouve, mais se trouvera réinterprété par les autres comme signe de la concupiscence, l'abaissement dans les plaisirs charnels de ce monde, alors que le seul accès à la divinité résiderait dans l'ascèse pour atteindre un Dieu hors de ce monde. Ce n'est pas le mot en lui-même qui possède un sens prédéterminé, mais l'ensemble du jeu d'associations créé par le système théorique au sein duquel il se situe qui lui offre des connotations recombinables selon les nécessités de la polémique. 
propre cadre de compréhension, convertissant la valeur positive du premier en valeur négative, et inversement.

On peut néanmoins reprendre à Maingueneau son analyse du processus de conversion sans y voir le signe d'une incompréhension radicale et indépassable entre les deux camps. Comme on l'a mentionné avec Bourdieu, s'il est possible d'exprimer un désaccord sur une question, cela signifie que l'on se trouve déjà d'accord sur les termes dans lesquels cette question se trouve posée ${ }^{26}$. À vrai dire, on peut trouver une conciliation entre la conception très pessimiste de la polémique comme dialogue de sourds chez Maingueneau, et celle d'un simple débat superficiel sur fonds de connivences dans les présupposés chez Bourdieu, si l'on a recours à la notion de «topos», exposée plus haut : dans un débat au sein duquel les interlocuteurs s'adressent l'un à l'autre, mais aussi (et surtout) à un public qu'ils doivent convaincre, ils doivent s'ancrer sur des associations d'idées et valorisations qu'ils estiment déjà répandues, et donc à même d'être utilisées stratégiquement pour y ancrer leurs propres propos. Les différents camps s'ancrent donc sur un topos (ou « lieu commun ») qu'ils tentent d'adapter à la thèse qu'ils défendent : si leur propre thèse parvient à se trouver identifiée à un topos, alors elle peut bénéficier, par contagion, de la valorisation dont jouit celui-ci.

Dans le cas des polémiques qui nous intéressent, on peut donc analyser des processus rhétoriques de conversion des valeurs "présent» (PR) et "passé » (PA). Chacune de ces deux valeurs peut se trouver convertie, par un camp ou par l'autre, d'une manière positive ou négative, selon la traduction opérée :

«Passé » peut ainsi se trouver converti en PA- pour désigner l'usage de l'opposant («désuet»), ou en PA+ pour désigner le sien propre («authentique »). De même, «Présent » peut se trouver converti en

26. Pierre Bourdieu (op. cit., p. 89) prend l'exemple des polémiques littéraires quant au «beau style» : pour discuter afin de déterminer, parmi plusieurs propositions concurrentes, ce qui constitue le «beau style», il faut encore partager, à titre de présupposé commun, l'idée qu'il existe un tel «beau style», et qu'il est possible de le déterminer. De même, concernant nos débats, nous avons déjà remarqué que les polémiques qui pourraient se formuler sous la forme de la question «Quelle forme de breton est la plus authentique ? " supposent déjà que l'application de la notion d'« authentique » à des usages linguistiques ait une signification. 
PR- pour désigner l'usage de l'opposant (« récent»), ou en PR+ pour désigner le sien propre («actuel»). Ainsi, le tout est de parvenir à se dire soi-même « authentique » sans être « désuet», et « actuel » sans être "récent », et ce pour chacun des deux camps, tout en parvenant également à retraduire les propos de l'opposant dans le sens opposé. Ce cadre d'analyse posé, on peut maintenant observer de quelle manière les différentes préfaces de dictionnaires construisent ces processus de conversion sémantique pour promouvoir un usage contre un autre.

\section{Application}

Il faudrait réserver un sort à part pour les productions du XVII au XIXe siècle, que l'on expliquerait en les resituant dans leur contexte historique spécifique ${ }^{27}$. Mais nous pouvons déjà noter que, chez Julien Maunoir, on trouve à la fois la mise en avant positive d'une passéité de la langue, qui passe alors par l'intermédiaire d'un rattachement au topos de la création divine («Tout ce qui est produit immédiatement de Dieu sans le secours des Creatures a vn eclat, qui surpasse en dignité les effects, a la production desquels les causes secondes \& instrumentales sont admises : c'est pour cette raison que [...] les langues primitiues [sont plus nobles] que celles qui ont esté inuentées par l'industrie des hommes $\left.{ }^{28} . »\right)$, et celle d'une actualité lorsqu'il s'agit pour lui de se justifier dans sa rénovation de l'orthographe, notamment l'abolition du $<$-ff $>$ pour les finales, et la notation des mutations consonantiques

27. À ce sujet, nous ne pouvons pour l'instant que renvoyer à la deuxième partie de notre thèse, en cours de rédaction. Il faudrait dégager deux grandes tendances dans ces époques : d'une part, le mouvement panceltiste, (incarné principalement par Maunoir, Grégoire de Rostrenen, Le Pelletier), qui, sous l'influence de Paul Pezron et John Davies, identifie toutes les langues d'Europe comme issues d'une racine "celtique ", dont le breton est alors décrit comme un vestige conservé. Les auteurs de cette époque se soucient rarement de modernité. D'autre part, un courant émergeant à partir du XIX (dont l'Essai sur l'bistoire de la langue bretonne, de La Villemarqué, pourrait être considéré comme le manifeste), que l'on qualifierait plutôt de « celtisme différentiel » au sens où les Celtes y sont mobilisés cette fois pour marquer une différenciation envers les Français, renvoyés depuis peu à des ancêtres gaulois ou romains. Ce serait avec Le Gonidec qu'apparaitrait la revendication de forger une langue adaptée aux besoins de la modernité.

28. Julien MAunoIr, Sacré Collège de Jésus, Quimper, Jean Hardouyn, 1659, p. 10. 
initiales ( Il semble qu'il est a propos de changer la façon ancienne ${ }^{29}$ des Escriuains Bretons, pour escrire le Langage Armorique, l'escriuant comme on le prononce »; "Qui est celuy qui pourra lire ces mots escrits à l'ancienne mode <ar Goaff> [...]»; «Les François depuis peu ont trouué cette façon d'escrire fort propre, escriuant comme ils prononcét $\left.{ }^{30} \cdot »\right)$.

Le discours celtomane insistera fortement sur la valorisation du passé, en érigeant les Celtes comme ancêtres fondateurs de tous les peuples européens, dont les Bretons seraient restés les purs représentants primitifs. On en trouve quelques exemples très explicites dans la préface que donne Charles Taillandier au dictionnaire de Louis Le Pelletier : "Cette Langue, la plus ancienne peut-être de celles que l'on parle aujourd'hui dans l'univers, nous conduit à la connaissance de nos origines; elle nous fait remonter jusqu'aux premiers habitants des Gaules, \& elle est elle-même le monument le moins équivoque de l'antiquité de la Nation Bretonne ${ }^{31}$. » L'usage de la notion de «Celtes » connaitra de forts remaniements au fil du XIXe siècle, notamment sous l'influence d'un contexte international qui voit émerger une « invention des traditions ${ }^{32}$ ", qui, au Pays de Galles, en Irlande, et en Écosse, mobilise la figure d'ancêtres imaginaires pour asseoir une nouvelle définition des appartenances locales se situant en opposition envers celles de la France ou de l'Angleterre ${ }^{33}$.

Sans qu'il soit possible ici de retracer la totalité de ces évolutions, on constate que la notion de «Celtes » est toujours invoquée par les lexicographes du $\mathrm{XX}^{\mathrm{e}}$, mais son contenu sémantique s'est modifié :

29. C'est nous qui soulignons, pour ce passage et pour tous les suivants.

30. Julien MAUnOIR, ibid., section « De l'Escriture».

31. Charles TAILlandiER, «Préface», Dictionnaire de la langue bretonne, où l'on voit son antiquité, son affinité avec les anciennes langues, l'explication de plusieurs passages de l'Écriture Sainte, et des auteurs profanes, avec l'etymologie de plusieurs mots des autres langues, Paris, François Delaguette, 1752, p. 1.

32. Eric HobSBAwm et Terence RAnger, The invention of tradition, Cambridge University Press, 1983, traduction française, Paris, Amsterdam, 2006.

33. Voir notamment Bernard TANGUY, Aux origines du nationalisme breton, Paris, Union Générale d'Édition, 1977 ; Simon JAMES, The Atlantic Celts. Ancient people or modern invention?, Londres, British Museum Press, 1999 ; Joseph RIO, Mythes fondateurs de la Bretagne. Aux origines de la celtomanie, Rennes, Ouest-France, 2000 ; Erwan CHARTIER-LE FlOCH, Histoire de l'interceltisme en Bretagne, Spézet, Coop Breizh, 2013. 
il ne s'agit plus de désigner un ancêtre commun, fondateur de toutes les populations d'Europe, mais surtout un ancêtre spécifique, c'est-àdire distinct de celui des Français. «Gaulois » et «Celtes », qui furent longtemps tenus pour synonymes, se trouvent maintenant distingués, et le fait que les Français se désignent désormais comme issus des premiers aboutit à ce que le second terme se trouve disponible pour les processus d'originations bretonnes.

\section{Les conversions dans le discours refondateur}

Cet ensemble de conceptions influence directement les choix des lexicographes de la tendance « refondatrice », qui décident, lorsque des besoins de créations lexicales se font sentir, de mettre en avant une parenté «celtique », et d'écarter les termes qui témoigneraient d'une influence du français :

"Les termes qui semblent influencés par le français ont aussi été laissés de côté, à moins qu'il n'y eût rien d'autre qui permettrait de nommer telle ou telle chose en breton [...] Nous avons dû plusieurs fois utiliser des néologismes pourtant et même former quelques termes. Sous ce rapport, nous avons décidé d'utiliser tout d'abord des termes ayant été attestés dans des états plus anciens de notre langue ou qui ont été empruntés à d'autres langues celtiques - au gallois principalement - ou encore des mots formés à partir d'éléments bretons ou celtiques dans l'ensemble ${ }^{34}$.»

Ce travail lexicographique put être présenté par les tenants de la tendance que nous avons nommée « refondatrice » comme une forme de correction par les intellectuels d'un certain laisser-aller lexicographique qui aurait mené les locuteurs de milieu populaire à adopter des formes influencées par le français :

«En Bretagne, depuis le début du XIXe siècle, une école de lettrés

34. Geriadur brę̧honeg gant skouerioù ha troiennoù, Le Relecq-Kerhuon, An Here, 1995, p. VIII. "Lezet eo bet ivez a-gostez an troiennoù a hañval bezañ levezonet gant ar galleg nemet ne vefe netra all a rofe tu da c'beriañ tra pe dra e brezhoneg. [...] Ranket hon eus ober meur a wežb gant nevezç'berioù koulskoude ha sevel un nebeud termenoù zoken. Er c'heñver-se hon eus dibabet implijout da gentañ-penn termenoù zo bet arveret e stadoù koshoc'b eus hor yezh pe zo bet amprestet digant yezhoù keltiek all - digant ar c'hembraek dreist-boll - pe c'boaz̧h gerioù zo savet diwar elfennoù brezhonek pe geltiek dre vras. » 
et de semi-lettrés groupés en associations et en académies œuvre à unifier et à purifier les parlers des illettrés, vestiges de la langue riche et raffinée du Haut Moyen Âge. De leurs efforts a résulté au second quart du XXe siècle une authentique littérature ${ }^{35}$. »

Si l'on perçoit donc de quelle manière cette démarche lexicographique est située par ses auteurs dans le cadre de valeurs $\mathrm{PA}+$, au sens où le recours aux « langues celtiques » et le travail pour « purifier» la langue lui permet de renouer avec un passé glorieux, et s'oppose donc à une évolution historique de la langue décrite comme une décadence subie sous l'influence du français, on percevra également, par le processus de conversion présenté, la tendance concurrente, celle que nous avons nommée « continuiste », pourra se trouver présentée sous la valeur PA-, comme manifestant une forme de nostalgie dépassée pour des usages anciens de la langue :

"[L'état de minorisation] fait l'enchantement des amateurs de langues "naturelles, authentiques" : il leur permet de faire des études phonologiques intéressantes, certes, mais qui ne servent en rien l'avenir des langues en question. [...] Les dictionnaires bretons les plus récents, dans leur partie bilingue français-breton, ont préféré laisser le terrain de la modernité en friche et s'en tenir au fonds ancien, comme si le monde était figé. Ils ont sciemment ignoré les travaux de néologie menés depuis près d'un demi-siècle. Les lexicographes ne peuvent fuir leurs responsabilités $[. .$.$] Toute attitude qui$ exclut la néologie et l'adaptation des termes internationaux de l'avenir de la langue bretonne la cantonne de facto au passé, la réduit à la portion congrue, lui interdit le vocabulaire nécessaire à dire le monde d'aujourd'hui, ce qui revient à la condamner à disparaittre d'elle-même ${ }^{36}$.»

C'est souvent dans un même mouvement que les protagonistes du débat peuvent opposer eux-mêmes leur valeur PR+ (adaptation à la modernité) à celle PA- qu'ils construisent discursivement chez leur opposant :

35. Guy ÉTIENNE, Geriadur ar bredelfennerez̧ e peder yez̧ : galleg, alamaneg, saozneg, brez̧oneg, Plomelin, Preder, 1983, p. XIII.

36. Martial MÉNARD, Dictionnaire français-breton, Quimper, Palantines, 2012, p. 10-11. 
«Ce dictionnaire breton est fait pour tous ceux qui mettent leur confiance dans l'avenir de la langue bretonne [...]. [...] il ne s'agit pas uniquement de la faire survivre, pas uniquement rester dans le même état - le parler chéri de nos chers anciens - met elle doit, si nous voulons qu'elle reste vivante, être la langue de création de tout ce qui naît, tout ce qui se forme, tout ce qui se crée dans l'esprit de n'importe quel brittophone qui souhaite faire usage de sa langue dans le monde d'aujourd'hui ${ }^{37}$. $»$

Comme nous l'avons dit plus haut, analyser ces phénomènes en termes de processus de conversion nous permet d'éviter de considérer comme contradictoires des discours qui, en même temps, revendiquent une «authenticité » et s'opposent à un "passéisme», revendiquent une dimension «actuelle » de la langue tout en s'opposant aux changements « récents ». Chaque interlocuteur met à profit, de la manière la plus opportune possible, les valeurs PR et PA pour parvenir à présenter sa propre démarche sous les valeurs $\mathrm{PR}+$ et $\mathrm{PA}+$, et celle du concurrent comme PR- et PA-. La néologie se trouve ainsi construite discursivement par ses promoteurs comme conciliant le meilleur de l'ancien (authenticité celtique) et du récent (adaptation aux besoins de la modernité), et les usages lexicaux concurrents, inversement comme ayant le pire de l'ancien (désuétude d'un vocabulaire resté traditionnel) et du récent (influence du français). On trouve par exemple dans une préface de Gwenhael Ar Menteg une articulation très nette entre les revendications d'actualité (" Autrefois $10 \%$ des gens habitaient en ville et les autres à la campagne, aujourd'hui on constate l'inverse. Autrefois le breton était une langue d'agriculteurs - alors très riche. Mais maintenant il y a besoin d'une langue citadine - tout aussi riche. Une langue du monde dans lequel nous vivons, une langue moderne que nous voulons utiliser ${ }^{38}$.») et d'authenticité ("La veuve de Loeiz Herrieu disait : "Ceux-là sont des Bretons Français, nous nous sommes des Bretons Celtes". Comprenez que les "Bretons Français" sont ceux

37. Geriadur brezhoneg, op. cit., p. xi, « Graet eo ar geriadur hollurezhonek-mañ evit an holl re a laka o fiziañ en amzer da zont ar brezhoneg [...]. [...] ned eo ket hepken dreistbevañ he deus d'ober, ned eo ket enta chom en he stad - lavar karet hor re gozh karet - met bez' e rank, mar fell dimp ma chomo bev, bezañ yez̧h krouiñ kement a c'han, kement a furm, kement a grou e spered nep brezhoneger a venn ober gant e yezh er bed a-vremañ. "

38. Alan MONFORT, Gerioù evit komz, bemdez, Vannes, Hiziv an Deiz, 2001, p. 4. 
qui utilisent des mots français, ou surnommés "internationaux" - le "breton abâtardisé" disait ma grand-mère -, et les "Bretons Celtes" ceux qui "ont envie que soit tirée des ressources de la langue la matière pour construire des néologismes”, c'est-à-dire ceux qui sont du côté de la langue bretonne bretonne, un breton qui ne soit pas un sabir ${ }^{39}$.»)

\section{Les conversions dans le discours continuiste}

Par opposition à ce premier courant lexicologique, on trouve un ensemble d'auteurs qui refuseront en partie la démarche de création néologique et privilégieront un breton collecté dans les usages oraux quotidiens des locuteurs que nous avons décrits comme «bretonnants »: Francis Favereau mentionne par exemple avoir voulu, dans son dictionnaire, «cerner ce breton vivant et populaire ", « un parler riche et réputé insaisissable», ou encore "photographier un usage authentique, qu'on savait déjà menacé à moyen terme », en constatant : « je ne trouvais guère cette verve dans les dictionnaires [...] où la norme écrite l'emportait par trop sur l'usage », et décrivant "le néologisme » comme «la maladie infantile du bretonnantisme ${ }^{40} »$. Il construit alors une opposition entre la «création intempestive de termes» et les termes «consacrés par un certain usage ». Cette opposition s'ancre dans des descriptions grammaticales précises puisqu'à la première dimension se trouve associée « cette abstraction ou cette nominalisation hypertrophiée », considérée comme « des formes linguistiques du " $\mathrm{mal}$ français" ", alors qu'à la seconde tendance correspond «la dérivation, naturelle en breton » ainsi que d'autres procédés (nominalisation des infinitifs, adjectivation des substantifs, adverbialisation des adjectifs) qui « correspond[ent] véritablement au génie de la langue bretonne et du celtique ${ }^{41} »$. L'objectif est donc là aussi présenté comme « donnant la priorité au breton authentique» sur des usages décrits comme artificiels, il s'agit de respecter «le caractère propre de notre cher breton de Bretagne, langue celte millénaire, en partie romanisée dans

39. Ibid. Le texte breton, non recopié ici, dit plus directement : «brez̧oneg bastard».

40. Francis FaVereau, Dictionnaire du breton contemporain, Morlaix, Skol Vreizh, 1992, p. V. Cette dernière expression renvoie à un essai de Lénine, intitulé Le gauchisme, maladie infantile du communisme.

41. Ibid. 
son vocabulaire, mais à la structure intacte et au style original que l'on sait ».

La critique provenant du courant opposé, selon laquelle « les termes français employés tels quels en breton » constitueraient la dénaturation récente d'une pureté lexicale originelle, subit elle aussi le processus de conversion : par un retournement habile, l'auteur oppose cet usage d'appropriations lexicales " pourtant authentiquement populaire et fort ancien, comme l'attestent les dictionnaires du XVIII" », qui «s'emploient donc en breton parlé, et ceci depuis des décennies, voire des siècles ", à « la norme édictée par des écrivains bretons inconnus hors de leurs cercles restreints », que "l'usage n'a jamais suivi», et qui se contente de «singer l'académisme d'outre-Couesnon qui est une autre forme du jacobinisme séculaire ${ }^{42}$ ! ». Ainsi, c'est le rejet des termes proches du français qui se trouve le plus associé à un raisonnement purement français, et donc sûrement blâmable à ce titre.

Symétriquement aux positions précédentes, on trouvera des discours qui considèrent l'adaptation de mots à partir du français ou de formes lexicales « internationales » comme témoignant d'une modernité $(\mathrm{PR}+)$, là où l'attachement à des racines celtiques se trouvera présenté comme une forme de passéisme :

"On imagine aussi assez mal un vocabulaire mathématique coupé de celui des langues de grande tradition scientifique. C'est pourquoi il nous semble clair que la création de vocabulaire mathématique breton ne peut se faire qu'en privilégiant les racines internationales, c'est-à-dire en s'appuyant sur le français, l'anglais, l'allemand et le russe. Toute autre voie nous apparait comme une voie de repli, ne pouvant conduire qu'à l'impasse dans le domaine scientifique ${ }^{43}$.»

Encore une fois, c'est la mobilisation des processus de conversion qui nous permet d'expliquer en quoi les discours qui valorisent à la fois une authenticité et une actualité, dans cette tendance, ne sont pas plus contradictoires que le précédents : dès lors que l'on s'intéresse

42. Ibid., p. VIII.

43. Jean MAROT, op. cit. [n. 1], p. 9. 
aux stratégies discursives mises en place dans la situation de polémique pour valoriser son propre propos, on perçoit que les valeurs PR et PA peuvent être tour à tour rendues positives ou négatives. C'est ainsi que Jean Marot, défendant le caractère actuel des formes linguistiques qu'il présente dans son dictionnaire des mathématiques, justifie également certains usages en invoquant leur ancienneté : "Le choix de somm = somme [...] est incontournable, d'autant plus qu'il est attesté en moyenbreton $^{44}$ (DBC). »; « on remarque que le verbe resoniñ appartient à la langue bretonne depuis Grégoire de $\operatorname{Rostren}^{45}$ (DBC) ».

Dans le discours continuiste également, les dimensions positives de l'actualité $(\mathrm{PR}+)$ et de l'authenticité $(\mathrm{PA}+)$ pourront donc se trouver entremêlées. Ainsi, dans la préface au dictionnaire Garnier, Pierre-Jakez Hélias mobilise un discours selon lequel les adaptations de termes identifiés comme « français » ne constituent ni une dégradation récente de la langue (PA-) ni une marque d'incapacité à s'adapter à la modernité (PR-), mais au contraire, le moyen pour pouvoir dire le monde d'aujourd'hui $(\mathrm{PR}+)$ tout en respectant un mode de raisonnement traditionnel $(\mathrm{PA}+)$ qui serait à ses yeux plus authentiquement breton que les néologismes celtiques. Cette articulation se trouve rendue possible par l'invocation d'une «créativité » propre aux locuteurs des « anciennes générations », douées d'un «sens inné de la langue » leur permettant de «créer à mesure» des tournures modernes tout en demeurant «typiques de l'esprit breton » :

« [...] les auteurs ont voulu combler certaines lacunes, particulièrement en ce qui concerne les mots abstraits et les vocabulaires techniques ou technologiques nouveaux. Les anciennes générations savaient les créer à mesure, mais l'usage de plus en plus répandu

\footnotetext{
44 Ibid., p. 21.

45 On pourrait se demander s'il y a lieu de situer Jean Marot dans la démarche « continuiste » comme nous l'avons fait. Si le choix de présenter un dictionnaire des mathématiques marque déjà un décalage avec ce que nous pourrions associer à cette démarche (puisque le vocabulaire très spécialisé que l'on y trouve n'est pas attesté auprès de locuteurs dits «natifs »), nous avons choisi de l'y ranger ici pour deux raisons : d'une part, la défense d'usages lexicaux proches du français contre la démarche de néologismes celtiques, d'autre part, les références fréquentes au dictionnaire de Francis Favereau. Néanmoins, le décalage de Marot envers les discours généralement tenus par la tendance continuiste rend son argumentation particulièrement intéressante par sa singularité : elle mérite une étude à part qui ne peut pas être proposée ici.
} 
du français a fait décliner cette créativité. Cependant, il est encore possible, avec du temps et de la patience à l'écoute des désignations pour des notions nouvelles et des objets nouveaux qu'un sens inné de la langue leur fait trouver spontanément et qui ne doivent rien à ces décoctions artificielles de racines. [...]

[...] le souci majeur de l'équipe rédactionnelle a été de multiplier les exemples sous forme de phrases ou d'expressions typiques de l'esprit breton et qui n'apparaissent pas comme des décalques du français. Le breton a de merveilleuses trouvailles qui n'ont rien de commun avec la langue de Descartes et les ressources de sa syntaxe sont d'une étonnante variété. Les plus anciens bretonnants de naissance reconnaittront ici leurs tournures originales et familières ${ }^{46}$ $[\ldots] »$.

\section{Conclusion}

Les préfaces de dictionnaires bretons fournissent donc un lieu privilégié pour l'observation de débats, eux-mêmes révélateurs des profonds changements qui ont affecté les conditions d'apprentissage et de pratique du breton au XXe, et notamment la polarisation en deux (ou trois) profils de locuteurs.

Nous avons tenté d'en proposer une analyse qui, du fait qu'elle s'est d'abord écartée délibérément du crédit théorique que l'on accorde généralement aux notions de « langue » ou d'« authenticité », peut ainsi se retourner sur l'usage discursif qui est fait de ces notions : il s'agit alors d'observer par quelles conversions une positivité des valeurs "présent» et "passé » peut se trouver invoquée ou revendiquée par chacun des camps. Dans l'analyse de tels processus, il ne nous appartient pas de trancher concernant la plus grande pertinence de l'une ou l'autre des propositions, l'objectif est bien plutôt d'analyser ce qu'elles nous révèlent de la situation linguistique bretonne dans son évolution historique.

Mais plus généralement, au-delà de la spécificité locale, l’application d'un tel cadre d'analyse à des discours sur la langue permet de

46. Dictionnaire breton, Paris, Garnier, 1986, p. 6-7. 
rappeler utilement certaines généralités : ce que l'on nomme «une langue », ou bien les conceptions répandues concernant ce qui est « un bon usage » de telle langue ou non, ne sont pas des données naturelles, mais les résultats de processus de définition. Ceux-ci nécessitent l'obtention d'une certaine reconnaissance par le public quant à la supériorité sur d'autres d'un usage que l'on tente de promouvoir : le travail historique des institutions pour produire la langue, et faire accepter telle ou telle définition des usages linguistiques à tenir pour légitime, est un travail toujours en cours, pas seulement par l'école de la République, mais aussi par des chercheurs-militants, engagés de bonne volonté dans des débats. Les linguistes, sociolinguistes, lexicographes, historiens de la langue, étymologistes, stylisticiens, etc., contribuent, par leur œuvre, à cette définition, dans la mesure où leur travail s'engage toujours sur l'obligation de promouvoir certains usages par rapport à d'autres (nous avons vu qu'un dictionnaire ne peut pas « tout » contenir), et où leurs convictions, elles-mêmes issues de leurs modes de socialisation linguistique, détermineront ce travail de définition de la langue. Par son caractère conflictuel, la situation de la lexicographie bretonne contemporaine nous offre un aperçu exacerbé de ces processus et nous permet de saisir clairement le rôle des descripteurs savants engagés dans la diffusion des valorisations et dévalorisations linguistiques, mettant au jour des processus moins visibles ailleurs.

L'étude des polémiques ne vise généralement pas à les résorber ; néanmoins, l'ambiguité constitutive de la situation étudiée ici, où les polémistes sont eux-mêmes des chercheurs, permet de suggérer une intégration des processus sociolinguistiques ici présentés dans les définitions linguistiques produites. Ainsi, la prise en compte de ces phénomènes par les acteurs de ces polémiques eux-mêmes, pourrait éventuellement permettre la formulation de nouvelles descriptions, où il serait possible de prendre en compte l'hétérogénéité des profils actuels de locuteurs comme une donnée déterminée par les évolutions historiques, plutôt que de se situer d'emblée dans un cadre conflictuel visant à invalider un usage concurrent. Plutôt que de tenter à tout prix de faire prévaloir comme plus légitime tel usage par rapport à tel autre, il deviendrait possible de se demander : « Pour quelles raisons, liées à mon contexte de socialisation linguistique, tiens-je en particulier à cet 
usage ? ", «Pour quelles raisons, liées à son contexte de socialisation linguistique, mon opposant tient-il en particulier à cet usage ? ", « Dans mes descriptions linguistiques, qu'est-ce qui relève d'une volonté de valoriser cet usage auquel je tiens, contre un autre ?». Bref, c'est à un appel à la réflexivité auquel la linguistique bretonne se trouve conviée ${ }^{47}$.

47. Bien entendu, cette réflexivité devrait commencer par s'appliquer à moi-même, qui devrais chercher quelles sont les conditions m'ayant dirigé vers une analyse des polémiques plutôt que vers une prise de position au sein de celles-ci. Le facteur générationnel, faisant que j’ai entendu parler de ces polémiques avant même d'être en mesure de me positionner à leur sujet, mon propre sentiment d'insécurité linguistique dans différents milieux où des descripteurs-savants-engagés trouvaient toujours une manière d'invalider comme "non-authentique» tel ou tel usage, sous des critères renouvelés selon les milieux, quels que soient les efforts, dans un sens ou dans un autre, pour se conformer aux codes de telle chapelle, et la diffusion de cadres théoriques issus de la sociolinguistique et de l'analyse de discours, qui permettent de dépassionner les débats en les départicularisant et en en offrant une approche analytique, constitueront sûrement trois bonnes pistes pour une telle analyse. 